\title{
Wide-spectrum slow magnetoacoustic waves in coronal loops
}

\author{
D. Tsiklauri and V. M. Nakariakov \\ Physics Department, University of Warwick, Coventry, CV4 7AL, England, UK
}

Received 25 July 2001 / Accepted 27 September 2001

\begin{abstract}
A model interpreting variations of EUV brightness upward propagating in solar coronal loops as slow magnetoacoustic waves is developed. A loop is considered to have a non-zero plane inclination angle and offset of the circular loop centre from the baseline. The model also incorporates effects of dissipation and gravitational stratification. A linear evolutionary equation is derived and applied to investigations of slow wave dynamics. Both the non-zero plane inclination and the offset decrease the growth of the wave amplitude due to stratification. It is shown that wide-spectrum slow magnetoacoustic waves, consistent with currently available observations in the low frequency part of the spectrum, can provide a rate of heat deposition sufficient to heat the loop. In this scenario, the heat would be deposited near the loop footpoints, which agrees with the current observational data.
\end{abstract}

Key words. magnetohydrodynamics (MHD) - waves - Sun: activity - Sun: corona - Sun: oscillations Sun: UV radiation

\section{Introduction}

Identification of possible mechanisms of heating of solar coronal plasma and acceleration of solar wind is among the unsolved problems of solar physics. In the last two decades there has been intensive theoretical work undertaken to advocate the idea of coronal plasma heating by magnetohydrodynamic (MHD) waves. In particular, it has been suggested that coronal loops could be heated by dissipation of high frequency slow magnetoacoustic waves (Porter et al. 1994a,b; Laing \& Edwin 1995). However, up until recent observational advances, these studies have been poorly substantiated because of the absence of observational evidence of MHD wave activity in the solar corona.

Recent observations performed with SOHO/EIT and TRACE imaging telescopes have established:

1. The existence of upward propagating perturbations of EUV brightness in long coronal loops at the bandpasses EIT $195 \AA$ (Berghmans \& Clette 1999) and TRACE $171 \AA$ (Berghmans et al. 1999; De Moortel et al. 2000), and EIT $195 \AA$ and TRACE $171 \AA$ simultaneously (Robbrecht et al. 2001). Probably, the same phenomenon was observed by Nightingale et al. (1999) in TRACE $171 \AA$ A.

Send offprint requests to: David Tsiklauri, e-mail: tsikd@astro.warwick.ac.uk
2 . The projected velocities of these plasma density perturbations are about $65-165 \mathrm{~km} \mathrm{~s}^{-1}$, which is below the sound speed corresponding to the temperature of the loop.

3 . The amplitude is usually $1-3 \%$ of the background density, reaching $5-6 \%$ in some observations.

4. The propagating disturbances are quasi-periodic with periods of 3-20 min. There is no well-defined correlation between the periods and physical parameters of the loops detected.

5. The disturbances are detected in the loop legs near footpoints and always propagate upwards. There are no observations of waves propagating downwards.

Two rival interpretations of the propagating disturbances have been suggested. In the first (Reale et al. 2000), the disturbances are considered as field-aligned flows of matter, generated by the siphon effect. In the second (Nakariakov et al. 2000), the propagating disturbances are associated with slow magnetoacoustic waves. The wavebased theory is similar to the model developed for the interpretation of compressive perturbations observed in polar plumes (Ofman et al. 1999; Ofman et al. 2000) in terms of slow magnetoacoustic waves. Both theories are consistent with the observations and with the MHD theory, but the flow-based model has difficulties explaining the periodicity and the symmetry of the front and back edges of the disturbance. Also, the observations do not show a change (acceleration) in the propagating disturbance speed, predicted by the flow-based theory. In contrast, the wavebased theory explains all the observational findings and, in 
particular, justifies the absence of downward-propagating disturbances.

In the wave-based theory (Nakariakov at al. 2000), it has been established that the main physical factors, which are important in the slow magnetoacoustic wave dynamics in long coronal loops, include: (i) gravitational stratification, which is responsible for wave amplitude amplification; (ii) dissipation, which leads to the conversion of wave kinetic energy into heat in the large wave numbers part of the spectrum, and (iii) nonlinearity, which generally yields wave front steepening by generation of higher harmonics, leading to enhanced dissipation. An evolutionary equation for the slow magnetoacoustic wave amplitude has been derived, which includes these three effects. However, in order to catch the physical essence of the problem without further complications, simple geometry of the loop, namely, zero inclination angle and zero offset of the centre from the solar surface has been assumed.

In contrast, observations show that the plane of a loop is not necessary perpendicular to the Sun's surface (a nonzero inclination) and the centre of the loop circle is usually situated below the surface (a non-zero offset) (e.g. Aschwanden et al. 1999). Consequently, a more elaborate theory of the slow magnetoacoustic waves in coronal loops, which includes the effects of the non-zero inclination and the non-zero offset, is required.

Soft X-ray and EUV coronal telescopes provide the possibility to determine the distribution of density, temperature and heat deposition along a loop. Priest et al. (1998), using Yohkoh/SXT observations, concluded that hot, soft X-ray loops were heated uniformly. Aschwanden et al. (1999), Aschwanden et al. (2000) and Aschwanden et al. (2001) analyzing SOHO/EIT and TRACE EUV observations argued that cooler loops were heated nonuniformly, near the loop footpoints. The specific mechanism for the energy deposition remains unknown and could, in principle, be associated with dissipation of slow magnetoacoustic waves.

De Moortel et al. (2000) have estimated the energy of slow waves observed to be insufficient to heat a loop. This result was based upon the assumption of the wave to be a single harmonic with a period of several minutes. However, currently available resolution of the telescopes does not allow us to detect periodic motions with periods shorter than a few minutes, even if they are present in the spectrum. On the other hand, the absence of a well-defined period in the loop waves suggests that their spectrum is probably continuous and not a single harmonic. We would like to point out that the main difficulty with the theory of coronal loop heating by dissipation of slow magnetoacoustic waves has been connected with the absence of observational evidence for these waves in the corona. It has been believed that the waves somehow generated below the temperature minimum are not able to penetrate through this layer. Now, observational evidence for long period longitudinal waves has been provided. The long period waves can have unresolved shorter period components in their spectrum. According to the theory, these short period spectral components dissipate very quickly, and deposit the energy in the near footpoint of the loop legs.

In this paper we further develop the model of Nakariakov et al. (2000) for one-dimensional slow magnetoacoustic waves in stratified coronal loops, taking into account the effects of the non-zero loop plane inclination angle and the semi-circular loop offset, and derive a generalized evolutionary equation for slow magnetoacoustic waves in coronal loops. It is shown that a coronal loop acts as a dissipative filter on the slow magnetoacoustic waves and that the short wavelength part of the wave spectrum dissipates in the nearest vicinity of the loop footpoints. By calculating the heat deposition rate, provided by the dissipation of the slow waves and comparing it with the observationally determined heat deposition rate in EUV loops, we show that sufficiently wide spectrum slow waves can deposit the required amount of energy in the lower parts of the loops, which is consistent with observations.

This paper is organized as follows: we formulate our generalized model in Sect. 2; Sect. 3 deals with the comparison of our model's predictions with the observations; and, finally, in Sect. 4 we close with a discussion of our main results.

\section{The model}

We consider a semi-circular loop of the curvature radius $R_{\mathrm{L}}$. The loop cross-section is taken to be constant. Validity of the latter assumption has been corroborated by Klimchuk et al. (1992) and by Aschwanden et al. (1999) for soft X-ray and EUV loops, respectively. The loop is filled with a gravitationally stratified magnetized plasma of constant temperature. Here, we generalize Nakariakov et al.'s (2000) work by incorporating effects of loop plane inclination and offset of circular loop centre from the baseline. This is necessary since observed loops have non-zero (measured from the normal to the solar surface) inclination angle $\alpha$ and non-zero offset of the circular loop centre from the coronal base line, $Z_{0}$, i.e. distance from the circle's centre to the solar surface. The sketch of the model geometry is shown in Fig. 1.

The gravitational acceleration projected on the loop tangential at any point which is located at a distance $s$ measured from the footpoint $(z=0$, in Fig. 1) along the loop, is

$g(s)=\frac{G M_{\odot}}{\left[R_{\odot}+h(s)\right]^{2}} \sin \theta(s) \cos \alpha$,

where $G$ is the gravitational constant, $R_{\mathrm{L}}$ is the loop radius, $R_{\odot}$ and $M_{\odot}$ are solar radius and mass, respectively, and $h$ is the distance from the point to the coronal base line. From simple geometric considerations (see Fig. 1) we deduct that $h(s)=\left[R_{\mathrm{L}}-Z_{0} \sec \theta(s)\right] \cos \theta(s) \cos \alpha$ and $\theta(s)=\arccos \left(Z_{0} / R_{\mathrm{L}}\right)-s / R_{\mathrm{L}}$. We then insert these two 


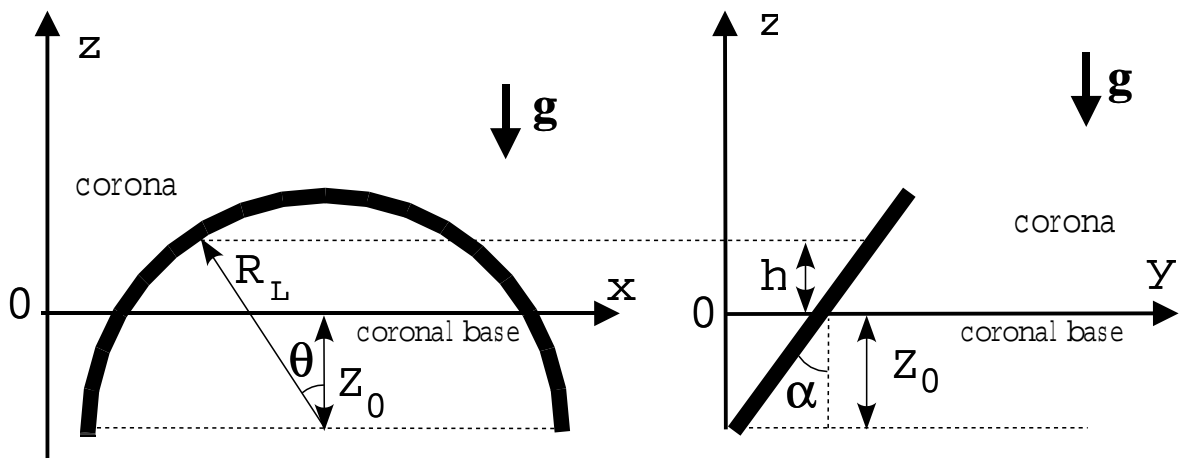

Fig. 1. The sketch of the model considered. A coronal loop is considered as a magnetic field line with density and gravitational acceleration varying along the axis of the cylinder.

functions into the expression for $g(s)$ and using trigonometric calculus, from which follows that

$\sin \theta(s)=\sqrt{1-\frac{Z_{0}^{2}}{R_{\mathrm{L}}^{2}}} \cos \frac{s}{R_{\mathrm{L}}}-\frac{Z_{0}}{R_{\mathrm{L}}} \sin \frac{s}{R_{\mathrm{L}}}$,

and

$\cos \theta(s)=\frac{Z_{0}}{R_{\mathrm{L}}} \cos \frac{s}{R_{\mathrm{L}}}+\sqrt{1-\frac{Z_{0}^{2}}{R_{\mathrm{L}}^{2}}} \sin \frac{s}{R_{\mathrm{L}}}$,

we therefore, obtain the final expression for $g(s)$

$$
\begin{aligned}
g(s)= & \frac{G M_{\odot}}{R_{\odot}^{2}}\left[\sqrt{1-\frac{Z_{0}^{2}}{R_{\mathrm{L}}^{2}}} \cos \frac{s}{R_{\mathrm{L}}}-\frac{Z_{0}}{R_{\mathrm{L}}} \sin \frac{s}{R_{\mathrm{L}}}\right] \cos \alpha \\
& \times\left[1+\cos \alpha \frac{R_{L}}{R_{\odot}}\left(\sqrt{1-\frac{Z_{0}^{2}}{R_{\mathrm{L}}^{2}}} \sin \frac{s}{R_{\mathrm{L}}}\right.\right. \\
& \left.\left.-\frac{Z_{0}}{R_{\mathrm{L}}}\left[1-\cos \frac{s}{R_{\mathrm{L}}}\right]\right)\right]^{-2}
\end{aligned}
$$

The loop length is considered to be the length of the arc between the footpoints (the points with $z=0$ in Fig. 1). The effect of the non-zero inclination angle and offset of the circular loop centre from the baseline can be summarized by analyzing Eq. (1). An increase of the inclination angle (starting from $\alpha=0$ ) deceases $g(s)$, which in turn results in less plasma density stratification. An increase in the loop centre offset leads to a decrease of the loop length and also decreases the angle between the loop leg and the surface of the Sun at the footpoint. The length of the loop is $L=2 R_{\mathrm{L}} \arccos \left(Z_{0} / R_{\mathrm{L}}\right)$. Obviously, the increase in $Z_{0}$ reduces the effective stratification of the plasma density in the loop.

The use of hydrostatic equilibrium equations and the isothermal equation of state allows us to write the stationary density profile along the loop as

$$
\rho_{0}(s)=\rho_{0}(0) \exp \left(-\frac{\gamma}{C_{s}^{2}} \int_{0}^{s} g\left(s^{\prime}\right) \mathrm{d} s^{\prime}\right),
$$

where $\gamma=5 / 3$ is the adiabatic index and $C_{\mathrm{S}}$ is the speed of sound. The temperature $T$ was assumed to be $1 \mathrm{MK}$, giving the sound speed $C_{\mathrm{s}}$ of $152 \mathrm{kms}^{-1}$.

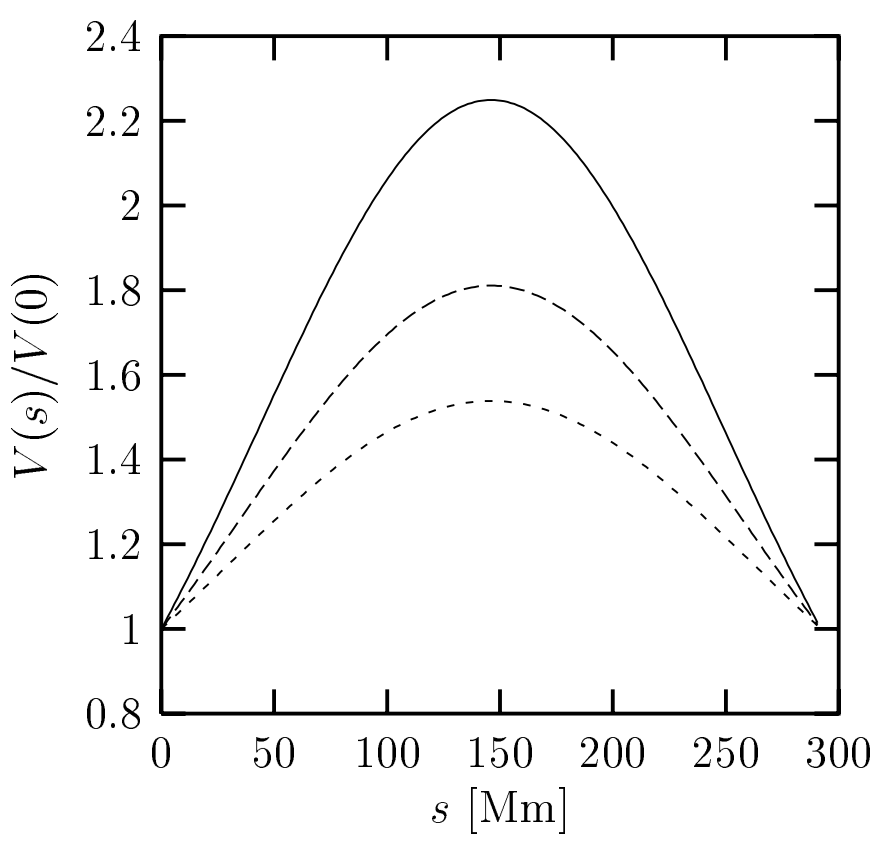

Fig. 2. Evolution of amplitude of a harmonic slow magnetoacoustic wave propagating along a coronal loop of the radius $93 \mathrm{Mm}$ and zero off-set for different loop plane inclination angles. The wave period is $300 \mathrm{~s}$. The medium is dissipationless, $\bar{\eta}=10^{-9}$. The solid curve corresponds to the inclination angle $\alpha=0$, and long and short dashed lines represent $\alpha=\pi / 4$ and $\alpha=\pi / 3$, respectively.

Inserting the generalized expression (1) for $g(s)$ into (2) we obtain

$\rho_{0}(s)=\rho_{0}(0) \exp \left[-\frac{\gamma g(0)}{C_{\mathrm{s}}^{2}}\right.$

$\times\left[R_{L} \sqrt{1-\left(\frac{Z_{0}}{R_{\mathrm{L}}}\right)^{2}} \sin \frac{s}{R_{\mathrm{L}}}-Z_{0}\left(1-\cos \frac{s}{R_{\mathrm{L}}}\right)\right] \cos \alpha$

$\times\left(1+\cos \alpha\left[\left(R_{\mathrm{L}} / R_{\odot}\right) \sqrt{1-\left(Z_{0} / R_{\mathrm{L}}\right)^{2}} \sin \left(s / R_{\mathrm{L}}\right)\right.\right.$

$\left.\left.\left.-\left(Z_{0} / R_{\odot}\right)\left(1-\cos \left(s / R_{\mathrm{L}}\right)\right)\right]\right)^{-1}\right]$. 


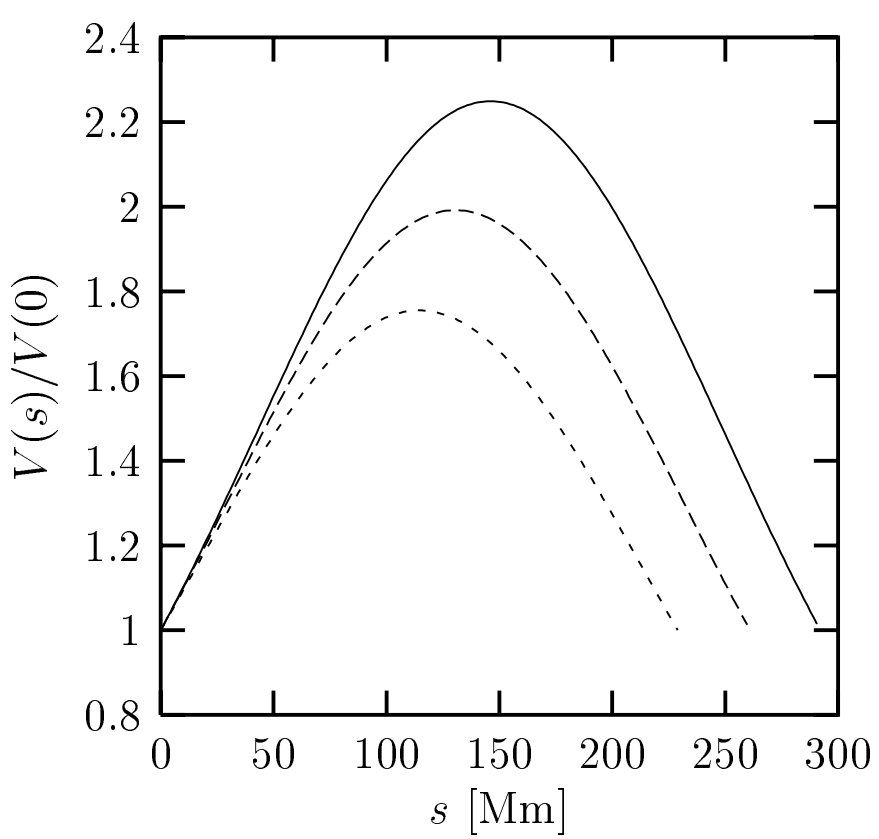

Fig. 3. Evolution of amplitude of a harmonic slow magnetoacoustic wave propagating along a coronal loop of the radius $93 \mathrm{Mm}$ and zero inclination angle. The wave period is $300 \mathrm{~s}$. The medium is dissipationless, $\bar{\eta}=10^{-9}$. The solid curve corresponds to the loop with the off-set $Z_{0}=0$, and long and short dashed lines represent $Z_{0}=R_{L} / 4$ and $Z_{0}=R_{L} / 2$ respectively.

Note that when $\alpha \rightarrow 0$, expressions (1) and (3) reduce to the case investigated by Nakariakov et al. (2000).

Assuming that the wavelength is much less than the scale height, the dissipation and the nonlinearity lengths, and applying the multi-scale expansion method, Nakariakov et al. (2000) obtained the evolutionary equation for weakly nonlinear, weakly dissipative slow magnetoacoustic waves in stratified coronal loops,

$$
\begin{aligned}
\frac{\partial V}{\partial s} & -\frac{1}{2 H(s)} V+\frac{\gamma+1}{2 C_{\mathrm{s}}} V \frac{\partial V}{\partial \xi} \\
& -\frac{1}{2 \rho_{0}(s) C_{\mathrm{s}}}\left[\frac{4 \eta_{0}}{3}+\frac{\kappa_{\|}(\gamma-1)^{2}}{\mathcal{R} \gamma}\right] \frac{\partial^{2} V}{\partial \xi^{2}}=0,
\end{aligned}
$$

where an effective scale height is introduced as $H(s)=$ $C_{\mathrm{s}}^{2} / \gamma g(s), \xi=s-C_{\mathrm{s}} t$ is the running coordinate, $\kappa_{\|}$ is the coefficient of thermal conduction, and $\eta_{0}$ denotes the volume viscosity. In the Eq. (4) term $V \partial V / \partial \xi$ can be safely neglected because it was shown that, for the amplitudes observed, the effect of the nonlinearity is insignificant. Moreover, neglecting the nonlinearity allows us to investigate solutions of the evolutionary equation in all the details analytically. Introducing dimensionless variables $\bar{R}_{\mathrm{L}}=R_{\mathrm{L}} / R_{\odot}, \bar{Z}_{0}=Z_{0} / R_{\odot}, \bar{\rho}=\rho_{0}(s) / \rho_{0}(0)$, $\bar{H}=H / R_{\odot}, \bar{V}=V / C_{\mathrm{s}}, \bar{s}=s / R_{\odot}, \bar{\xi}=\xi / R_{\odot}$ and the normalized coefficient of dissipation as

$$
\bar{\eta}=\frac{1}{\rho_{0}(0) C_{\mathrm{s}} R_{\odot}}\left[\frac{4 \eta_{0}}{3}+\frac{\kappa_{\|}(\gamma-1)^{2}}{\mathcal{R} \gamma}\right]
$$

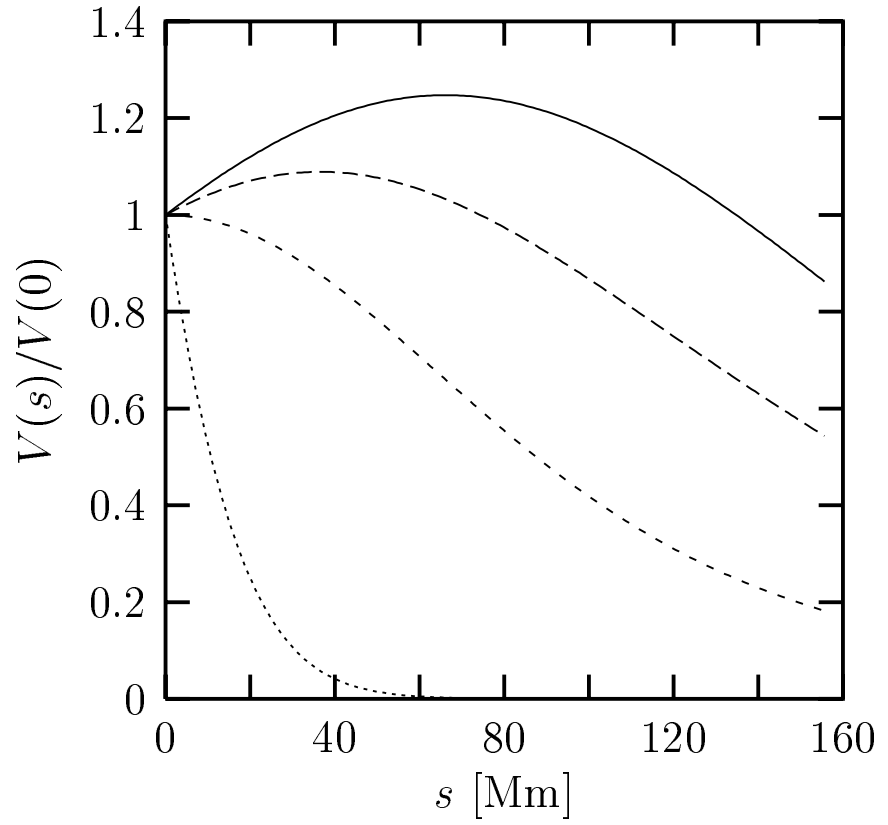

Fig. 4. Evolution of the slow wave amplitude in the average EUV loop (Aschwanden et al. 1999) with the parameters: $R_{\mathrm{L}}=93 \mathrm{Mm}, Z_{0}=62 \mathrm{Mm}, \alpha=7^{\circ}$. The normalized coefficient of dissipation $\bar{\eta}$ is $4.0 \times 10^{-4}$. The solid curve corresponds to the wave period of $600 \mathrm{~s}$, while long dashed, short dashed and dotted curves correspond to $300 \mathrm{~s}, 120 \mathrm{~s}$ and $60 \mathrm{~s}$ respectively.

and neglecting the non-linear term in Eq. (4) we finally obtain

$\frac{\partial \bar{V}}{\partial \bar{s}}-\frac{1}{2 \bar{H}(\bar{s})} \bar{V}-\frac{\bar{\eta}}{2 \bar{\rho}(\bar{s})} \frac{\partial^{2} \bar{V}}{\partial \bar{\xi}^{2}}=0$.

Equation (6) together with (1) and (3) generalizes the linear part of the equation derived by Nakariakov et al. (2000) to the case of non-zero loop plane inclination and the offset, $Z_{0}$.

Considering a harmonic wave, $\propto \exp (i k \xi)$, we solve Eq. (6) for the amplitude of the $k$ th spectral component,

$\bar{V}(\bar{s}, k)=\bar{V}(0, k) \exp \left[\phi_{1}(\bar{s})-k^{2} \phi_{2}(\bar{s})\right]$,

where

$\phi_{1}(\bar{s})=\int_{0}^{\bar{s}} \frac{1}{2 \bar{H}\left(\bar{s}^{\prime}\right)} \mathrm{d} \bar{s}^{\prime}=\frac{\gamma g(0)}{2 C_{s}^{2} R_{\odot}}$

$\times\left[\bar{R}_{\mathrm{L}} \sqrt{1-\left(\frac{\bar{Z}_{0}}{\bar{R}_{\mathrm{L}}}\right)^{2}} \sin \frac{\bar{s}}{\bar{R}_{\mathrm{L}}}-\bar{Z}_{0}\left(1-\cos \frac{\bar{s}}{\bar{R}_{\mathrm{L}}}\right)\right] \cos \alpha$

$\times\left(1+\cos \alpha\left[\bar{R}_{L} \sqrt{1-\left(\bar{Z}_{0} / \bar{R}_{\mathrm{L}}\right)^{2}} \sin \left(\bar{s} / \bar{R}_{\mathrm{L}}\right)\right.\right.$

$\left.\left.-\bar{Z}_{0}\left(1-\cos \left(\bar{s} / \bar{R}_{\mathrm{L}}\right)\right)\right]\right)^{-1}$

$\phi_{2}(\bar{s})=\int_{0}^{\bar{s}} \frac{\bar{\eta}}{2 \bar{\rho}\left(\bar{s}^{\prime}\right)} \mathrm{d} \bar{s}^{\prime}$

and $k$ is the dimensionless wave number, $k=2 \pi R_{\odot} / \lambda=$ $2 \pi R_{\odot} /\left(C_{\mathrm{S}} P\right)$ with $\lambda$ and $P$ being the wavelength and 
wave period respectively. Here, we used the fact that short wavelength slow magnetoacoustic waves are practically dispersionless and described by the dispersion relation $\omega \approx C_{\mathrm{s}} k$. Consequently, their spatial and temporal spectra are similar.

The parametric studies of solution (7) are shown in Figs. 2 and 3. Here, the loop radius was chosen to be the same as the average EUV loop determined by Aschwanden et al. (1999). Figure 2 demonstrates the behaviour of a harmonic wave in identical loops with different values of the inclination angle $\alpha$. Naturally, the increase of the inclination angle decreases the value of the maximum amplitude the wave can reach. Indeed, a larger inclination angle means less stratification of the plasma density in the loop, therefore lower maximal values. Figure 3 shows the evolution of the wave amplitude along the loop for different offsets of a circular loop centre from the baseline, $Z_{0}$. The increase in the loop centre offset leads to the decrease of the loop length and also decreases the angle between the loop leg and the surface of the Sun at the footpoint. The length of the loop is $L=2 R_{\mathrm{L}} \arccos \left(Z_{0} / R_{\mathrm{L}}\right)$. Obviously, the increase in $Z_{0}$ reduces the effective stratification of the loop density and this results in lower maximal amplitudes reached by the waves.

\section{Evolution of the spectrum}

Equation (7) describes evolution of the spectral component $k$. In the exponent, the first term is positive in the ascending stage of the wave propagation, becomes zero when the wave reaches the loop apex and then becomes negative. The second term is always negative. Consequently, in the ascending stage, the wave evolution is described by the competition of these two terms describing effects of stratification and dissipation, respectively. Importantly, these two mechanisms operate in different spectral domains. Mathematically, Eq. (7) is similar to an evolutionary equation of a dissipative filter in the theory of radio circuits. Therefore, a coronal loop acts on slow magnetoacoustic waves as a dissipative filter. If we knew the initial spectrum of the wave, the equation would allow us to determine the spectrum at each given height $s$ of the loop. Unfortunately, the initial spectrum, especially its short wavelength part, cannot be determined with the current resolution of EUV telescopes.

Evolution of the spectrum is accompanied by the dissipation of the spectral components. Different spectral components have different effective scale heights of the dissipation. Assuming the initial wave spectrum and following the spectrum evolution along the loop, we can determine the energy deposition rate at each given height $s$. Indeed, this function can be tested against the observations.

In order to compare predictions of our model with the observational data, we have investigated the behaviour of different slow magnetoacoustic wave spectral components in the average EUV loop determined by Aschwanden et al. (1999), with $R_{\mathrm{L}}=93 \mathrm{Mm}, Z_{0}=62 \mathrm{Mm}, \alpha=7^{\circ}$, corresponding to the loop length of about $L=156 \mathrm{Mm}$.

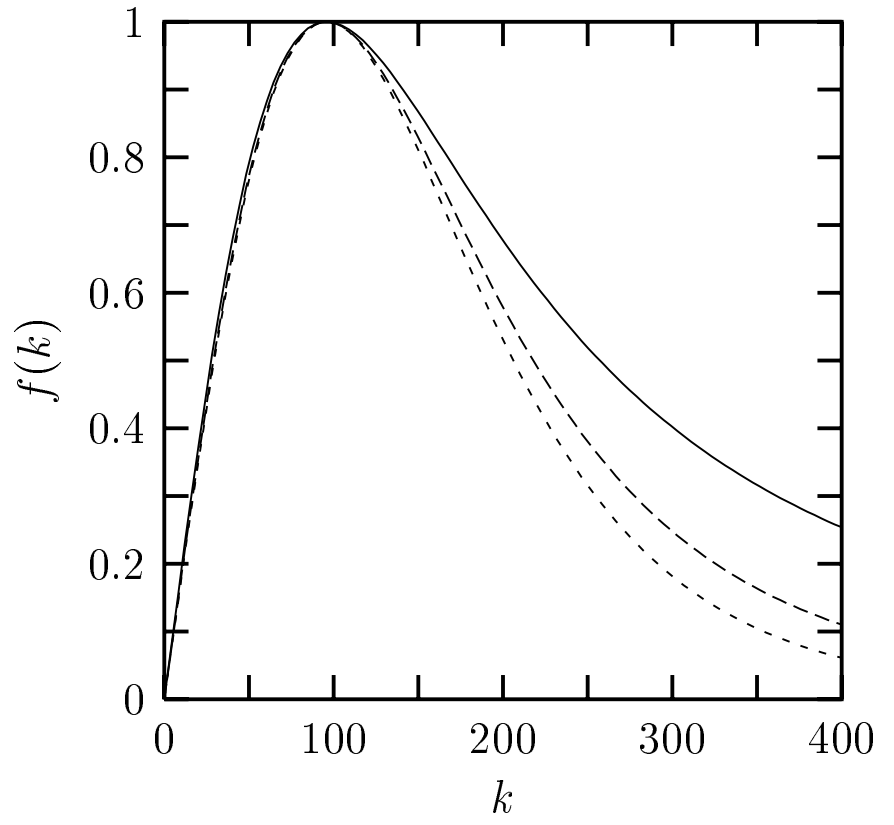

Fig. 5. Possible initial spectra $f(k)$ of slow magnetoacoustic waves. The solid curve corresponds to $n=3$, and long and short dashed curves to $n=5$ and 7 , respectively. The dimensionless wave number $k$ is connected with the wavelength $\lambda$ as $k=2 \pi R_{\odot} / \lambda$.

The evolution of different spectral components is shown in Fig. 4. The dissipative coefficient $\bar{\eta}$ has the same value as was estimated in Nakariakov et al. (2000). The spectral components corresponding to shorter wavelengths (i.e. shorter period) dissipate faster and closer to the loop footpoint.

The initial spectrum of the slow waves, $\bar{V}(0, k) \equiv$ $\sqrt{f(k)}$ is unknown, but it should satisfy certain criteria. According to observations, the spectrum should have a maximum in the vicinity of $180-420 \mathrm{~s}$. The velocity amplitude of the maximum is about $1-3 \%$ of the speed of sound. Perhaps, there are also maxima in the shorter period part of the spectrum, but they have not been resolved observationally. So, we assume that the spectrum gradually declines with the wave period. In order to avoid singularities in the integration of the dissipated wave energy over the whole spectrum $k=[0,+\infty)$, we assume that $f(k) \rightarrow 0$ when $k \rightarrow+\infty$ and $k \rightarrow 0$. Thus, we choose the spectrum $f(k)$ with a single maximum at $k_{*}$. A function which satisfies these requirements is

$$
f(k)=n^{n / 2}\left(\frac{a}{2}\right)^{(n-1) / 2} k\left(\frac{n-1}{2} a+k^{2}\right)^{-\frac{n}{2}}(n \geq 3) .
$$

The spectra with $n=3,5,7$ are shown in Fig. 5. For any $n$, the spectrum $f(k)$ attains its maximal value at $k_{*}=\sqrt{a / 2}$. For $a=1.84 \times 10^{4}, k_{*} \approx 96$, which corresponds to a wave period of $300 \mathrm{~s}$, and $n=3$, the actual spectral peak $(f(k)>0.69)$ is situated between the wave numbers corresponding to 1.5 and 12 min periods, which is consistent with observations. Note that $f(k) \propto k^{-(n-1)}$ asymptotically as $k \rightarrow+\infty$. The energy contained in the spectrum is determined by the spectral 
index $n$. Higher values of the spectral index correspond to narrower spectra.

The wave energy flux dissipated into heat in the loop segment from the footpoint to the distance $s$ is defined as

$F_{\mathrm{D}}(s)=\int_{0}^{+\infty}\left[F_{\mathrm{D} k}(0, k)-F_{\mathrm{D} k}(s, k)\right] \mathrm{d} k$,

where

$F_{\mathrm{D} k}(s, k)=\frac{1}{2} \rho_{0}(s) \bar{V}^{2}(0, k) A^{2} C_{\mathrm{s}}^{3} \exp \left[2 \phi_{1}(s)-2 k^{2} \phi_{2}(s)\right]$

and $A$ is the amplitude of the strongest harmonic in the spectrum, measured in the units of the sound speed $C_{\mathrm{s}}$, and Eq. (7) was used. To evaluate the integral in Eq. (11), we use the spectrum (10) and expressions (8) and (9). For the odd integer $n$, the integrals can be evaluated analytically, in particular

$\int_{0}^{+\infty} F_{\mathrm{D} k}^{n=3}(s, k) \mathrm{d} k=\frac{1}{2} \rho_{0} A^{2} C_{\mathrm{s}}^{3}(1.5 \sqrt{3} a)$

$\times\left(a^{-1 / 2}-\sqrt{2 \pi \phi_{2}(s)} \mathrm{e}^{2 \phi_{2}(s) a}\left[1-\operatorname{erf}\left(\sqrt{2 a \phi_{2}(s)}\right)\right]\right)$

$\int_{0}^{+\infty} F_{\mathrm{D} k}^{n=5}(s, k) \mathrm{d} k=\frac{1}{2} \rho_{0} A^{2} C_{\mathrm{s}}^{3}\left(5^{5 / 2}(a / 2)^{2} / 3\right)$

$\times\left((2 a)^{-3 / 2}+2 \sqrt{\pi}\left(2 \phi_{2}(s)\right)^{3 / 2} \mathrm{e}^{2 \phi_{2}(s)(2 a)}\right.$

$\left.\times\left[1-\operatorname{erf}\left(\sqrt{(2 a) 2 \phi_{2}(s)}\right)\right]-2\left(2 \phi_{2}(s)\right)(2 a)^{-1 / 2}\right)$

and

$\int_{0}^{+\infty} F_{\mathrm{D} k}^{n=7}(s, k) \mathrm{d} k=\frac{1}{2} \rho_{0} A^{2} C_{\mathrm{s}}^{3}\left(7^{7 / 2}(a / 2)^{3} / 15\right)$

$\times\left(3(3 a)^{-5 / 2}-4 \sqrt{\pi}\left(2 \phi_{2}(s)\right)^{5 / 2} \mathrm{e}^{2 \phi_{2}(s)(3 a)}\right.$

$\times\left[1-\operatorname{erf}\left(\sqrt{(3 a) 2 \phi_{2}(s)}\right)\right]-2\left(2 \phi_{2}(s)\right)(3 a)^{-3 / 2}$

$\left.+4\left(2 \phi_{2}(s)\right)^{2}(3 a)^{-1 / 2}\right)$.

Here, $\operatorname{erf}(x)=(2 / \sqrt{\pi}) \int_{0}^{x} \exp \left(-t^{2}\right) \mathrm{d} t$ is the error function. In the evaluation of the integrals, we use that $\rho_{0}(s) \exp \left[2 \phi_{1}(s)\right]=1$ and

$$
\begin{aligned}
I(n=3)= & \int_{0}^{+\infty} \mathrm{e}^{-\mu x} / \sqrt{(x+a)^{3}} \mathrm{~d} x \\
= & 2 / \sqrt{a}-2 \sqrt{\pi \mu} \mathrm{e}^{a \mu}(1-\operatorname{erf}(\sqrt{a \mu})), \\
I(n=5)= & \int_{0}^{+\infty} \mathrm{e}^{-\mu x} / \sqrt{(x+2 a)^{5}} \mathrm{~d} x \\
= & \frac{2}{3}\left((2 a)^{-3 / 2}+2 \sqrt{\pi} \mu^{3 / 2} \mathrm{e}^{a \mu}\right. \\
& \left.\times(1-\operatorname{erf}(\sqrt{a \mu}))-2 \mu(2 a)^{-1 / 2}\right),
\end{aligned}
$$

$$
\begin{aligned}
I(n=7) & =\int_{0}^{+\infty} \mathrm{e}^{-\mu x} / \sqrt{(x+3 a)^{7}} \mathrm{~d} x \\
& =\frac{2}{15}\left(3(3 a)^{-5 / 2}-4 \sqrt{\pi} \mu^{5 / 2} \mathrm{e}^{a \mu}(1-\operatorname{erf}(\sqrt{a \mu}))\right. \\
& \left.-2 \mu(3 a)^{-3 / 2}+4 \mu^{2}(3 a)^{-1 / 2}\right) .
\end{aligned}
$$

The observationally determined rate of heat deposition at a position $s$ of the loop can be approximated as

$E_{\mathrm{H}}(s)=E_{\mathrm{H} 0} \exp \left(-s / s_{\mathrm{H}}\right)$,

where $E_{\mathrm{H} 0}$ is the heating rate at the footpoint and $s_{\mathrm{H}}$ is the heating scale height. The specific values of $E_{\mathrm{H} 0}$ and $s_{\mathrm{H}}$ were determined by Aschwanden et al. (1999) for EIT loops and by Aschwanden et al. (2000, 2001) for TRACE loops. A physical value identical to $F_{\mathrm{D}}(s)$ introduced in this paper, can be constructed by integration of Eq. (19) over $s$,

$F_{\mathrm{D}}^{\mathrm{obs}}(s) \equiv \int_{0}^{s} E_{\mathrm{H}}(s) \mathrm{d} s=E_{\mathrm{H} 0} s_{\mathrm{H}}\left(1-\mathrm{e}^{-s / s_{\mathrm{H}}}\right)$.

When $s=L / 2$, at the loop apex, the value of the function $F_{\mathrm{D}}^{\text {obs }}(s)$ is the total energy power introduced by Aschwanden et al. (2001, Eq. (12)).

The comparison of the theoretical dependences of the wave energy flux dissipated into heat in a loop segment from the footpoint to the position $s$ given by Eqs. (13), (14) and (15) and the observationally determined dependence (20) is shown in Fig. 6. Here, we use the parameters of nonuniform heating determined in (Aschwanden et al. 2001), $E_{\mathrm{H} 0}=10^{-3.0 \pm 0.3} \mathrm{erg} \mathrm{cm}^{-3} \mathrm{~s}^{-1}$ and $s_{\mathrm{H}}=12 \pm 5 \mathrm{Mm}$. The normalized dissipation is $\bar{\eta}=4.0 \times 10^{4}$. The density of plasma near the loop footpoint, which corresponds to the concentration at the base of the average loop, $n_{0}=1.92 \times 10^{9} \mathrm{~cm}^{-3}$, is $\rho_{0}=4.1 \times 10^{-15} \mathrm{~g} / \mathrm{cm}^{3}$. The amplitude $A$ of the wave, corresponding to the peak in the spectrum (10), is taken to be $A=0.03$, i.e., $3 \%$ of the sound speed. The observational curve has the error bars determined by choosing plus and minus signs in the $E_{\mathrm{H} 0}$ and $s_{\mathrm{H}}$, given above.

For $n=3$ the theoretical curve $F_{\mathrm{D}}(s)$ fits the median value for the $F_{\mathrm{D}}^{\mathrm{obs}}(s)$ well. The curve corresponding to a steeper spectrum, with $n=5$, also marginally fits the observational results. Bearing in mind a relatively large magnitude of the observational errorbars, steeper spectra $(n>5)$ do not fit the data. This is a simple manifestation of the fact that a too steep spectrum does not provide enough energetics to heat the loop to the observed value.

\section{Discussion}

Recently observed variations of EUV emission intensity, propagating upward along the legs of solar coronal loops, are interpreted satisfactorily in terms of the slow magnetoacoustic waves. We generalized the model of the slow wave evolution in a loop, suggested by Nakariakov et al. (2000) by taking into account effects of loop plane inclination and offset of the semi-circular loop centre from the 


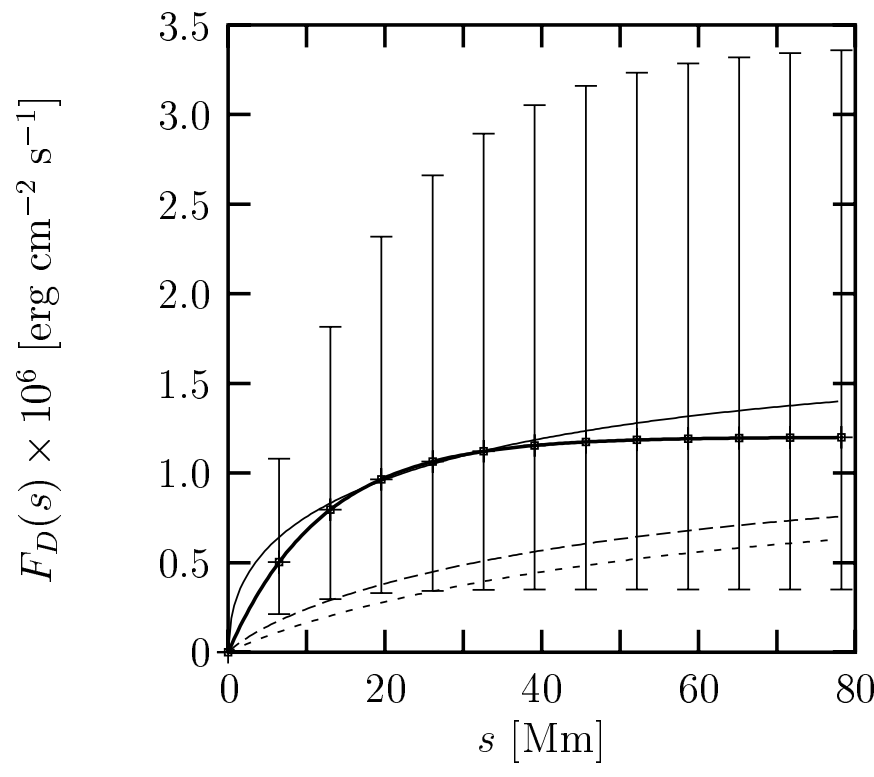

Fig. 6. The slow magnetoacoustic wave energy flux dissipated into heat in a loop segment from the footpoint to the position $s$ in comparison with the observationally determined nonuniform heating of an average loop. The thin solid curve corresponds to the spectral index $n=3$, the long dashed curve to $n=5$ and short dashed curve to $n=7$. The observational dependence is the thick solid curve with the error bars.

solar surface. An evolutionary equation which governs the wave propagation in the presence of gravitational stratification and dissipation was derived. Both non-zero inclination and offset affect the slow wave evolution, reducing the stratification of the loop plasma and, consequently, the amplitude growth of the upward propagating waves.

It was found that coronal loops act as dissipative filters of slow magnetoacoustic waves. Different spectral components have different dissipation lengths and the wave spectrum evolves with height or with the distance along the loop. Therefore, the amount of wave energy deposited in the loop decreases with the height and the wave dissipation deposits the energy in the lower parts of the loops. Making certain assumptions about the spectrum of the waves near the loop footpoints, we constructed a function describing the wave energy flux dissipated into heat in a loop segment. This function was compared with an observationally determined function approximating the nonuniform heat deposition requirements. We conclude that the observed heat deposition into coronal loops can be interpreted as dissipation of slow magnetoacoustic waves, provided the wave spectrum is sufficiently wide. This conclusion is consistent with previously obtained results by Porter et al. (1994a,b), but is based upon recently obtained observational information on the actual existence and parameters of slow magnetoacoustic waves in coronal loops and on the nonuniform distribution of heat deposition in EUV loops. In the scenario proposed, the short wavelength components of the slow wave spectrum, with short dissipation lengths, are filtered out in the loop legs, depositing the energy and heating the loops near the footpoints, while the long wavelength spectral components, with longer dissipation lengths, propagate further and are detected by EUV telescopes.

Strictly speaking, a realistic spectrum of slow magnetoacoustic waves is restricted by the acoustic cutoff wave number from below and by the inverse mean free path length from above. However, this does not seem to affect the results obtained, as the impact of the long wavelength (lower frequency) part of the spectrum to heating is weak and as the short wavelength slow waves are efficiently dissipated in the collisionless regime too, depositing energy near loop footpoints.

Therefore, the theoretical results on slow wave dissipation are consistent with the phenomenological results on EUV coronal loop heating and with the observed amplitude of the waves in the long wavelength part of the spectrum. The wide spectrum slow magnetoacoustic waves can, indeed, deposit a sufficient amount of energy near the loop footpoints, which is in agreement with the observations of Aschwanden et al. (1999, 2000, 2001). We would like to emphasize that this possibility is purely theoretical due to the not yet fully observed spectrum of the slow magnetoacoustic waves. However, our results indicate that the slow waves cannot be excluded from consideration as a possible mechanism for heating of the coronal loops.

Acknowledgements. The authors would like to thank Markus Aschwanden and Erwin Verwichte for valuable discussions. DT acknowledges financial support from PPARC Postdoctoral Fellowship.

\section{References}

Aschwanden, M. J., Newmark, J. S., Delaboudiniere, J.-P., et al. 1999, ApJ, 515, 842

Aschwanden, M. J., Nightingale, R. W., \& Alexander, D. 2000, ApJ, 541, 1059

Aschwanden, M. J., Schrijver, C. J., \& Alexander, D. 2001, ApJ, 550, 1036

Berghmans, D., \& Clette, F. 1999, Sol. Phys. 186, 207

Berghmans, D., Clette, F., Robbrecht, E., \& McKenzie, D. 1999, ESA SP-448, 575

De Moortel, I., Ireland, J., \& Walsh, R. W. 2000, A\&A, 355, L23

Klimchuk, J. A., Lemen, J. R., Feldman, U., Tsuneta, S., \& Uchida, Y. 1992, PASJ, 44, L181

Laing, G. B., \& Edwin, P. M. 1995, Sol. Phys., 161, 269

Nakariakov, V. M., Verwichte, E., Berghmans, D., \& Robbrecht, E. 2000, A\&A, 362, 1151

Nightingale, R. W., Aschwanden, M. J., \& Hurlburt, N. E. 1999, Solar Phys. 190, 249

Ofman, L., Nakariakov, V. M., \& Deforest, C. E. 1999, ApJ, 514,441

Ofman, L., Nakariakov,V. M., \& Sehgal, N. 2000, ApJ, 533, 1071

Porter, L. J ., Klimchuk, J. A., \& Sturrock, P. A. 1994a, ApJ, 435,482

Porter, L. J., Klimchuk, J. A., \& Sturrock, P. A. 1994b, ApJ, 435,502

Priest, E. R., Foley, C. R., Heyvaerts, J., et al. 1998, Nature, 393, 545

Reale, F., Peres, G., Serio, S., et al. 2000, ApJ, 535, 423

Robbrecht, E., Verwichte, E., Berghmans, D., et al. 2001, A\&A, 370,591 